\title{
INFEZIONI RECENTI DA HIV: VALUTAZIONE DELL'INDICE DI AVIDITÀ ANTI - HIV CON UN TEST COMBINATO PER HIV Ag E Ab
}

\author{
Bossi V.'; Galli C. ${ }^{2}$ \\ 'Virologia, ospedale Amedeo di Savoia, ASL3, Torino; \\ ${ }^{2}$ Medical Marketing, Abbott Diagnostici, Roma.
}

Introduzione. La valutazione dell'avidità anticorpale è un parametro utilizzato come ausilio nella diagnosi di molte infezioni virali recenti. La misurazione dell'indice di avidità (AI) degli anticorpi anti-HIV, di cui è stata già dimostrata la semplicità ed accuratezza, è stata inizialmente effettuata con un test automatizzato di terza generazione per anticorpi antiHIV1/2. In questo studio abbiamo voluto verificare se è possibile applicare questa procedura anche con un test di "quarta generazione", che consente la rilevazione associata di antigene p24 e di anticorpi anti-HIV.

Metodi. Sono stati analizzati 240 campioni consecutivi di routine da soggetti con nuova diagnosi di infezione da HIV. Il siero è stato prediluito in due aliquote, rispettiva-mente con guanidina- $\mathrm{HCl} 1 \mathrm{M}$ e con tampone MEIA, che sono state analizzate in singolo lo stesso giorno con due test automatizzati su piattaforma Abbott AxSYM, uno per soli anticorpi (AxSYM HIV1/2gO) e uno per p24 e anticorpi (AxSYM $\mathrm{HIV} \mathrm{Ag/Ab} \mathrm{Combo).} \mathrm{Per} \mathrm{ogni} \mathrm{campione} \mathrm{è} \mathrm{stato} \mathrm{valutato} \mathrm{l'AI}$ (rapporto tra il segnale rispetto al valore soglia $(\mathrm{S} / \mathrm{CO})$ tra il campione trattato con guanidina e quello trattato con tampone) e sono stati confrontati, da un punto di vista qualitativo e quantitativo, i risultati ottenuti con i due metodi. La precisione della procedura con il test Combo è stata valutata con un protocollo NCCLS su repliche multiple di 10 campioni. Risultati. La imprecisione totale dell'AI con il test AxSYM Combo, è risultata inferiore all'11\% (media: 6,93+2,40). In ragione di questa variabilità, per il confronto qualitativo tra $i$ due metodi è stato adottato un valore soglia di $\mathrm{AI}<0,75$ per le infezioni recenti (meno di 6 mesi) e $\geq 0,85$ per le infezioni non recenti, con una zona grigia (GZ) tra 0,75 e 0,84 . La concordanza tra $\mathrm{i}$ due test è risultata del $90,4 \%$ considerando tutti i risultati, e del 97,6\% escludendo i campioni GZ (22, pari al $9,2 \%$, con il test per soli anticorpi; 18 , pari al 7,5\% con il test Combo). Diversi risultati discordanti erano dovuti alla presenza di Ag p24 con un WB negativo o indeterminato. La correlazione tra i valori di AI ottenuti con i due metodi era elevata $(r=0,88)$. I nostri dati preliminari suggeriscono che è possibile valutare l'AI anti-HIV anche con i test combinati per HIV Ag e Ab, in ragione dell'efficiente rappresentazione degli epitopi immunodominanti di gp41 con questi ultimi. 